REVIEW

\title{
Heliox for asthma in the emergency department: a review of the literature
}

\author{
A D Reuben, A R Harris
}

Emerg Med J 2004;21:131-135. doi: 10.1136/emj.2002.003483

January 2002 saw the relaunch by BOC of Heliox, a gaseous mixture of helium and oxygen, for the use in a wide range of respiratory conditions. Despite a lapse of over 65 years since it was first used, and a large number of studies and case reports advocating its use, it remains an enigma, its use sporadic, and its role undefined. This paper reviews the discovery of helium and early medical use of helium oxygen mixtures and outlines areas where Heliox already has confirmed benefit as well as one or two areas that are currently under investigation. It will also look specifically at the use of Heliox in acute exacerbations of asthma and perform a thorough review of the current literature.
See end of article for authors' affiliations

Correspondence to:

Dr A Reuben, Emergency Department, Bristol Royal Hospital for Children, Paul $\mathrm{O}^{\prime}$ Gorman Building, Bristol BS2 8BJ, UK'; adamreuben@hotmail.com

Accepted for publication 18 June 2003
$\mathrm{H}$

elium was first isolated from the mineral Clevite in 1895. Its use in place of nitrogen as a carrier gas for oxygen was first described by Barach in the early 1930s. ${ }^{1}$ It was recommended as an adjunct to the treatment of respiratory failure, in particular for obstructive lesions of the larynx, trachea, and airways. Barach championed the use of helium oxygen mixtures as first line agents in the management of acute exacerbations of asthma, and this did gain some early favour in the absence of other recognised or effective forms of treatment. Enthusiasm for its use tailed off in the 1940s for two main reasons. The first was the onset of the second world war when main sources of helium were lost but more significant was the subsequent development of potent bronchodilators and mucolytic agents that proved far more effective. ${ }^{2}$ Interest in Heliox resurfaced in the early 1980s when deaths from asthma began to rise $^{3}$ but evidence of beneficial action is sparse and conflicting, though more recent case reports and clinical trials have suggested a benefit in selected patients.

\section{MECHANISM OF ACTION}

Helium is a colourless tasteless, non-combustible, non-explosive gas that is seven times lighter than air. ${ }^{4}$ One of its most important properties is that it is biologically inert and insoluble in human tissues having no bronchodilator or anti-inflammatory effect. Its key benefit seems to be as a temporising agent working while conventional treatments have time to act. ${ }^{5}$ Helium has the lowest specific gravity of any gas with the exception of hydrogen, which is highly combustible. Low specific gravity is associated with low density, and this is proportional to the flow rate of the gas, the lower the density, the higher the rate of flow. Combining helium and oxygen gas (Heliox) results in a gas with a similar viscosity to air but with a substantially lower density. ${ }^{6}$

To understand how Heliox may be of benefit in various disorders of the airways, it is necessary to be familiar with a few concepts of physics.

Gas flow in airways may be laminar, turbulent, or a combination of the two (transitional). Laminar flow is the most efficient way in which oxygen is delivered to the more distal parts of the bronchial tree. The type of flow occurring at any given point is determined by the Reynolds number of the gas. This is a unitless quantity that is proportional to the product of the airway diameter and the velocity and density of the gas, divided by its viscosity. ${ }^{7}$ When the Reynolds number is high (greater than 4000) flow is predominantly turbulent, when the Reynolds number is low (less than 2000) flow is principally laminar. The effective cross sectional area of the airways increases with each division of the airways from the trachea to bronchi-as this happens the flow rate of inspired gas falls. As the flow rate drops, the Reynolds number falls and flow becomes more laminar than turbulent. Within the substance of the lungs, there will be a transitional region where turbulent flow becomes laminar. Exercise, or any disease that increases ventilatory requirements will shift this transitional area distally causing a greater predominance of turbulent flow. ${ }^{4}$

Heliox acts by lowering the resistance to gas flow within the airways and permitting an increase in ventilation. This it does for two main reasons. Firstly, and most importantly, breathing Heliox leads to a reduction in the Reynolds number, converting turbulent flow into more efficient laminar flow. Secondly, because of its low density, Heliox decreases the pressure gradient needed to achieve a given level of turbulent flow and this in theory reduces the work of breathing. The use of Heliox in asthma and upper airways obstruction is not for the treatment of the underlying disease, but is used to reduce airways resistance and respiratory muscle work until definitive treatments act. ${ }^{3}$

Anecdotal evidence suggests that to be effective at reducing airways resistance by significant amounts the concentration of helium must be high, ideally greater than $70 \%$ of the inhaled gas mixture. This will limit the amount of oxygen that can be delivered simultaneously. However, the hypoxaemia associated with airways disease is usually modest and is usually responsive to 
small amounts of oxygen because the underlying mechanism of gas exchange impairment is a ventilation perfusion mismatch. ${ }^{7}$

\section{SAFETY}

Because helium is an inert gas that has no effect on human metabolism, it can be safely used in most patients. Review of the available literature reveals little evidence of any adverse reactions for patients breathing Heliox. Patients with high oxygen requirements may not tolerate mixtures containing high concentrations of helium, although in all of the studies performed to date, there is only one reported case of a patient having to be withdrawn on the grounds of hypoxia. ${ }^{20}$ Helium has a high thermal conductivity and may be associated with a lowering of body temperature when used for prolonged periods, therefore a patients temperature should be monitored during prolonged periods of administration. ${ }^{5}$ Overall Heliox seems to be safe and well tolerated in the mixtures used to treat disorders of the upper airways.

\section{ADMINISTRATION AND TECHNICAL INFORMATION}

To use Heliox does not require the introduction of any additional equipment beyond that which is available in most emergency departments as standard. Commercial mixtures are available in portable cylinders containing 1200 litres of gas, which is ideal for storage and transport within the emergency department. Standard cylinders contain Heliox in a mixture of $79 \%$ helium and $21 \%$ oxygen, though nasal cannulas can be used to provide additional oxygen to the patient receiving Heliox where there is a risk of hypoxia. However, as the oxygen concentration increases, the gas mixture becomes denser and its clinical effectiveness is reduced. ${ }^{5}$ Administration is best via a non-rebreathing facemask as this allows the best concentration to be delivered to the patient without any mixing of room air to occur. ${ }^{6}$ Heliox cylinders cost about four times as much as an equal volume of oxygen and this has no doubt contributed to some of the reluctance to its use and to carrying out large scale randomised controlled trials. This cost however, must be balanced against potential for reduction in morbidity and mortality associated with various airway disorders and specifically the cost associated with intubation and mechanical ventilation.

\section{USES OF HELIOX Upper airways obstruction}

Upper airways obstruction is the most common indication for which Heliox is used. The literature supporting its use was reviewed by Smith and Buros, ${ }^{8}$ who reported that the greater the severity of the obstruction, the more dramatic the benefit from breathing Heliox. In contrast with widely held beliefs, they also suggested that Heliox could be of benefit at comparatively low concentrations of helium $(\mathrm{FiHe}<0.6)$, concluding that it is a valuable but under used intervention. Many published case series and reports have described the rapid and dramatic response to inhaled helium oxygen mixtures in patients with upper airways obstruction with improvement in respiratory distress that often averts the need for intubation. Patients become less anxious, more comfortable and more cooperative as the work of breathing is decreased. ${ }^{9}$ There is also growing support for the use of Heliox as an adjunct to ventilation in cases of iatrogenic upper airway obstruction such as bronchoscopy. ${ }^{4}$

\section{COPD}

The benefits of Heliox in COPD are less clearly reported, although there is on-going research into its use in place of oxygen in acute exacerbations. Sidwa investigated the effects of breathing Heliox in 15 patients with severe exacerbations of COPD who were acutely short of breath. ${ }^{10}$ After only 15 minutes of breathing Heliox they noted a modest reduction in $\mathrm{PaCO}_{2}$ with a significant reduction in functional residual capacity. This they argued indicated a significant reduction in intrinsic PEEP. Other studies have shown that Heliox consistently improves both inspiratory and expiratory flow producing a reduction in dynamic hyperinflation with a consequent improvement in gas exchange. ${ }^{4}$ Reduction in hyperinflation improves mechanical advantage by increasing respiratory muscle efficiency and decreasing the work of breathing. Decreased work of breathing will reduce the volume of carbon dioxide produced. ${ }^{5}$ Rodrigo et al studied the available literature in their review for the Cochrane library. ${ }^{11}$ They looked at four studies, two of which were based on nonventilated patients and two that looked at patients being mechanically ventilated. They managed to obtain data for only two of the studies. They concluded that current evidence is insufficient to support the use of helium-oxygen mixtures in the treatment of acute exacerbations of COPD, but state that future randomised controlled trials would be of value.

\section{Croup}

Duncan et al performed some of the earliest work looking at the use of helium and oxygen mixtures in croup, treating seven children with severe croup refractory to epinephrine with Heliox 70:30. ${ }^{12}$ They found that all patients showed a significant reduction in their croup scores with none requiring intubation.

Weber et al in 1998 performed a study comparing the additive effect of a helium oxygen mixture on racemic epinephrine on croup scores in 29 children with moderate to severe croup treated with humidified oxygen and corticosteroids. ${ }^{13}$ All patients received cool humidified oxygen and intramuscular dexamethasone before being randomly assigned to receive either Heliox or racemic epinephrine. They found that both Heliox and racemic epinephrine were associated with improvements over time, concluding that both led to similar improvements. It is on the basis of this study that Heliox is used as part of routine protocols for the management of refractory childhood croup in many departments.

\section{Asthma}

Status asthmaticus is defined by Bechler Karsch (1994) as an asthma attack that is refractory to conventional treatment and can lead to respiratory failure and death if not properly managed. ${ }^{14}$ The vast majority of patients with acute exacerbations of asthma are successfully treated with routine therapy. A small subset of patients will however, fail to respond to conventional therapy and may require urgent intubation and mechanical ventilation. ${ }^{9}$ The initiation of mechanical ventilation in patients with severe asthma may save lives, but is associated with increased morbidity. Anecdotal case studies have suggested that there is a small subgroup of patients with status asthmaticus, unresponsive to conventional therapies that may benefit from breathing Heliox until definitive therapies take hold. ${ }^{15}$ As previously noted helium is an inert agent and Heliox has no bronchodilator or anti-inflammatory effect, its main action seems to be in acting as a temporising agent allowing the usual forms of therapy to reach their peak activities.

Shiue and Gluck treated 10 patients with status asthmaticus and respiratory acidosis with a mixture of Heliox (25\%$40 \%$ oxygen) in addition to conventional treatment. ${ }^{2}$ Most patients sensed a rapid decrease in their dyspnoea within the first few minutes after the inhalation of the Heliox mixtures, 9 of 20 patients had reduction of their $\mathrm{PaCO}_{2}$ within 20 minutes of breathing Heliox with a corresponding rise in $\mathrm{pH}$. All patients showed noticeable improvement after one hour. The authors recognise that separation of the effects 
produced by bronchodilators and Heliox is not possible, but conclude that the relation is at least synergistic, with an initial failure of response to conventional treatment.

Kass and Castriotta performed a study of 12 patients with respiratory acidosis secondary to acute exacerbations of asthma presenting to the emergency department. ${ }^{16}$ All of the patients were treated with Heliox either 70:30 or 60:40 depending on the level of hypoxia. Seven patients received Heliox via a non-rebreathing facemask, five patients through a mechanical ventilator. They found that eight patients responded to Heliox therapy (response being defined as drop in $\mathrm{PCO}_{2}$ to a normal level, or by $>15 \%$, along with a rise in $\mathrm{pH}$ of >0.05). The remaining four patients showed some improvement in $\mathrm{pH}$ and $\mathrm{PCO}_{2}$, but not enough to enter the previously defined category. They concluded that Heliox could lead to an acute improvement in respiratory acidosis in patients with severe asthma. They recognised that the lack of control subjects again raised the possibility that improvements represented the delayed effects of previously administered treatments, but suggested that the rapidity of response when breathing Heliox indicates a direct beneficial relation. Interestingly the authors attempted to define which group of patients were most likely to benefit from intervention with Heliox, reporting that those patients with a shorter duration of symptoms were more likely to respond.

Kass and Terregino performed the first randomised controlled trial of Heliox (70:30 helium:oxygen) compared with $30 \%$ oxygen in acute exacerbations of asthma. ${ }^{17}$ They included 23 adult patients with clinically significant exacerbations of asthma as defined by PEFR of $<200 \mathrm{l} / \mathrm{min}$. All patients received conventional treatment as per departmental protocols (intravenous methylprednisolone, nebulised albuterol) but were randomised to receive either Heliox (11 patients) or oxygen (12 patients) if there was no clinical improvement and PEFR remained $<200 \mathrm{l} / \mathrm{min}$. Arterial blood gas examination revealed that all patients involved in the study had a normal $\mathrm{pH}$. Nine of those in the treatment group had a greater than $25 \%$ improvement in PEFR at 20 minutes compared with only two in the control group. They found also that treatment with Heliox was responsible for a rapid reduction in subjective dyspnoea as compared with the control group. This was the first randomised controlled trial to show that the reported benefits of Heliox, which had been suggested for some time, were in fact real. There was

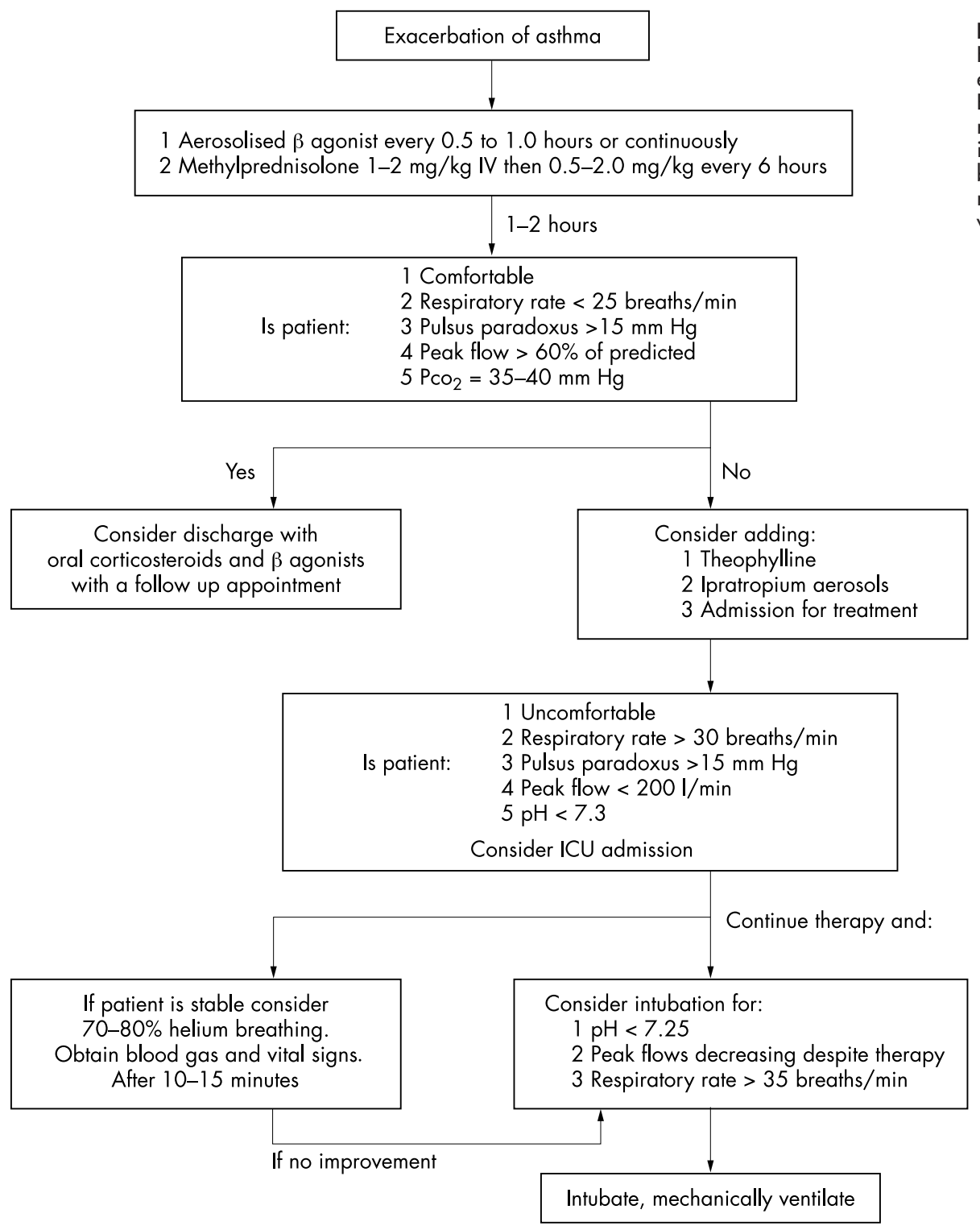

Figure 1 Algorithm for the use of Heliox in the treatments of exacerbations of asthma as used in Bridgeport Hospital, Connecticut. They recommend Heliox for the most severely ill patients not responding to routine bronchodilator treatments but not yet requiring intubation and mechanical ventilation. 
however much criticism of these results. There is a school of thought widely held that suggests that patients with rapid onset exacerbations of asthma exhibit a more severe picture clinically at presentation but at the same time have a more rapid recovery as compared with slow onset episodes. Both of these studies are congruent with this idea with patients apparently responding to Heliox having a significantly shorter duration of symptoms.

Manthous et al administered an 80:20 helium oxygen mixture to 29 patients presenting to the emergency department with acute exacerbations of asthma defined by PEFR $<50 \mathrm{l} / \mathrm{min}$ and pulsus paradoxus of $>15 \mathrm{~mm} \mathrm{Hg}$ after conventional treatments. ${ }^{18}$ They found a reduction in pulsus paradoxus in 15 of 16 patients breathing Heliox that was significantly greater than during air breathing and an increase in PEFR that was also significantly greater than in control subjects breathing air. They argued that because pulsus paradoxus indirectly represents inspiratory muscle work, Heliox actually reduces the work of breathing in patients with acute severe asthma.

Not all of the literature favours the use of Heliox in asthma. Dorfman et al carried out a prospective randomised controlled trial comparing the use of 80:20 helium:oxygen mixture with air in addition to continuous nebulised albuterol and ipratropium in 40 patients. ${ }^{19}$ They found no significant difference between the treatment and control groups in terms of changes in baseline characteristics, except for heart rate that increased after the study intervention. There was no significant difference in the change in respiratory rate, systolic and diastolic pressures, oxygen saturations, and PEFR. The lack of any perceived benefit may reflect the exclusion of those patients with the most severe exacerbations of asthma. In this study the authors included patients with PEFR of $<70 \%$ of best or predicted with no reference to the proportion whose exacerbations fell into the categories of severe or life threatening, although those patients deemed to need intubation or unable to give informed consent were excluded. It is highly probable based on the evidence of previous studies and case reports, that it is this group of patients who would receive most benefit from treatment with Heliox as a temporising agent to defer the need for intubation while conventional therapies have additional time to act.

Henderson et al also carried out a randomised controlled trial of Heliox therapies in patients with exacerbations of asthma presenting to the emergency department. ${ }^{20}$ They found no benefit conferred from the addition of Heliox to a standard asthma regimen in emergency department patients with mild to moderate exacerbations of asthma.

Verbeek and Chopra studied the effect of breathing a 70:30 helium oxygen mixture on $\mathrm{FEV}_{1}$ in patients presenting with acute exacerbations of asthma. ${ }^{21}$ Unlike previous studies that included conventional therapies, they looked solely at the use of Heliox as a treatment option on its own for the management of acute exacerbations of asthma. They looked at a sample of 13 patients presenting with moderate to severe exacerbations of known asthma $\left(\mathrm{FEV}_{1} 20\right.$ to $60 \%$ of predicted) and treated them with five minutes of Heliox (70:30) via a non-rebreathable mask having measured baseline $\mathrm{FEV}_{1}$. They excluded all patients who had received any bronchodilator therapy within one hour of arrival in the emergency department or whose saturations fell below $90 \%$ at any time as measured by pulse oximetry. There was no demonstrable improvement in absolute $\mathrm{FEV}_{1}$ or percentage predicted $\mathrm{FEV}_{1}$. They found that there were more subjects who showed either a slight decline or no change in $\mathrm{FEV}_{1}$ than those who showed a positive response. The authors are quick to recognise criticisms of their own study, suggesting that the group of patients that they studied stood little to gain from Heliox therapy because their airway obstruction was not severe. Previous work has also suggested that at least $70 \%$ of helium is required in a gaseous mixture to receive the full advantage of its physical properties. While this study presents valuable criticism of the role of Heliox in the management of acute exacerbations of asthma, its results need to be viewed in context. With the advent and defined role of potent bronchodilators, the value of Heliox is likely to be as an adjunct to conventional therapies, acting as a temporising agent while conventional therapies act. Heliox on its own has no bronchodilator or anti-inflammatory effect and is therefore unlikely to influence the course of an asthma episode.

The most substantial literature review and the most recent body of evidence was performed by Rodrigo et al in their review in the Cochrane library. ${ }^{3}$ They looked at all of the studies involving randomised, single, or double blind trials to determine the effect of adding Heliox to standard medical care in the course of acute asthma as measured by pulmonary function and clinical end points. A total of four randomised controlled trials were selected for inclusion, four involving adults and one paediatric study. On the basis of these trials they concluded that the existing evidence does not provide support for the administration of helium oxygen mixtures to patients presenting to the emergency department with moderate to severe acute asthma, but recognised that further research is warranted in the form of randomised controlled trials. Recent guidelines published for the management of acute exacerbations reflect this uncertainty and the use of Heliox in acute adult asthma is not recommended on the basis of the present evidence. ${ }^{22}$

Despite the lack of concrete evidence to support its use in asthma, Heliox still forms an integral part of the treatment algorithms for exacerbations of asthma in some centres. Figure 1 shows how Heliox fits into the protocol for the management of asthma at Bridgeport Hospital, Connecticut. ${ }^{9}$ They recognise that most patients are successfully treated with routine therapy but that there are a small subset of patients who require urgent intubation and ventilation. There also exists a small population of patients with status asthmaticus who fail to respond to routine therapy but still have respiratory muscle reserve. It is this second group who they may benefit from breathing Heliox until definitive therapies take hold. Patients remain under close observation until there are signs of significant improvement and may still require intubation if they deteriorate.

\section{CONCLUSION}

Despite a lapse of over 60 years since Barach first described the use of helium-oxygen mixtures for the management of obstructive disorders of the respiratory tract, its use remains sporadic and undefined. The physical properties of helium make it an ideal agent for use as a carrier gas for oxygen in place of nitrogen for various conditions affecting the respiratory tract. The low density and viscosity of Heliox helps to promote greater and smoother gas flow, decrease airway resistance, and decrease the work of breathing in selected patients. There is a body of evidence to support its use in parallel with conventional forms of treatment in asthma, COPD, croup, and a variety of other respiratory conditions, although further work will be required to define its precise role.

\section{Authors' affiliations \\ A D Reuben, A R Harris, Emergency Department, Royal Devon and} Exeter Hospital, Exeter, Devon, UK

\section{REFERENCES}

1 Barach AL. The use of helium in the treatment of asthma and obstructive lesions in the larynx and trachea. Ann Intern Med 1935;9:739-65. 
2 Shive MD, Gluck EH. The use of helium-oxygen mixtures in the support of patients with status asthmaticus and respiratory acidosis. J Asthma 1989;26:177-80.

3 Rodrigo G, Rodrigo C, Pollack C, et al. Helium-oxygen mixtures for nonintubated acute asthma patients. Cochrane Library. Issue 4. Oxford: Update Software, 2001.

4 Ball JAS, Rhodes A, Grounds RM. A review of the use of helium in the treatment of acute respiratory failure. Clinical Intensive Care 2001;12:105-13.

5 McGee DL, Wald DA, Hinchcliffe S. Helium-oxygen therapy in the emergency department. J Emerg Med 1997;15:291-6.

6 Browne-Heitschmidt MG, Cassidy JB. Heliox: a new treatment for lifethreatening asthma. Pediatric Nursing 1997;23:479-82.

7 Madison JM, Irwin RS. Heliox for asthma, a trial balloon. Chest 1995; 107:597-8.

8 Smith SW, Buros M. Relief of imminent respiratory failure from upper airway obstruction by use of helium-oxygen: case series and brief review. Acad Emerg Med 1999;6:953-6.

9 Manthous CA, Morgan S, Pohlman A. Heliox in the treatment of airflow obstruction: a critical review of the literature. Resp Care 1997;42:1034-42.

10 Sidwa DM, Montenegro HD, Goldman MD, et al. Helium-oxygen breathing in severe chronic obstructive pulmonary disease. Chest 1985;87:790-5.

11 Rodrigo G, Pollack C, Rodrigo C, et al. Heliox for the treatment of exacerbations of chronic obstructive pulmonary disease. Cochrane Library. Issue 1. Oxford: Update Software, 2003.
12 Duncan PG. Efficacy of helium-oxygen mixtures in the management of severe viral and post-intubation croup. Can J Anaesth 1979;26:206-12.

13 Weber JE, Chudnofsky CR, Younger JG, et al. A randomized comparison of helium-oxygen mixture (Heliox) and racemic epinephrine for the treatment of moderate to severe croup. Pediatrics 2001;107:E96.

14 Bechler-Karsch A. Assessment and management of status asthmaticus. Pediatric Nursing 1994;20:217-23.

15 Austan F. Heliox inhalation in status asthmaticus and respiratory acidaemia: a brief report. Heart Lung 1996;25:155-7.

16 Kass JE, Castriotta RJ. Heliox therapy in acute severe asthma. Chest 1995; 107:757-60.

17 Kass JE, Terregino CA. The effect of heliox in acute severe asthma: a randomized controlled trial. Chest 1999;116:296-300.

18 Manthous CA, Hall JB, Melmed A. Heliox improves pulsus paradoxus and peak expiratory flow in nonintubated patients with severe asthma. Am J Resp Crit Care Med 1995; 151:310-14.

19 Dorfman TA. Inhaled heliox does not benefit ED patients with moderate to severe asthma. Am J Emerg Med 2000;18:495-7.

20 Henderson SO, Acharya P, Kilaghbian T. Use of heliox driven nebuliser therapy in the treatment of acute asthma. Ann Emerg Med 1999;33:141-6.

21 Verbeek PR, Chopra A. Heliox does not improve FEV1 in acute asthma patients. J Emerg Med 1998;16:545-8.

22 thoracic Society. British guideline in the management of asthma. Thorax 2003;58(suppl 1). 